\title{
Single-shot spatiotemporal measurements of high-field terahertz pulses
}

J. van Tilborg, C. B. Schroeder, Cs. Tóth, C. G. R. Geddes, E. Esarey, and W. P. Leemans Lawrence Berkeley National Laboratory, University of California, Berkeley, California 94720, USA

\section{DISCLAIMER}

This document was prepared as an account of work sponsored by the United States Government. While this document is believed to contain correct information, neither the United States Government nor any agency thereof, nor the Regents of the University of California, nor any of their employees, makes any warranty, express or implied, or assumes any legal responsibility for the accuracy, completeness, or usefulness of any information, apparatus, product, or process disclosed, or represents that its use would not infringe privately owned rights. Reference herein to any specific commercial product, process, or service by its trade name, trademark, manufacturer, or otherwise, does not necessarily constitute or imply its endorsement, recommendation, or favoring by the United States Government or any agency thereof, or the Regents of the University of California. The views and opinions of authors expressed herein do not necessarily state or reflect those of the United States Government or any agency thereof or the Regents of the University of California. 


\title{
Single-shot spatiotemporal measurements of high-field terahertz pulses
}

\author{
J. van Tilborg, C. B. Schroeder, Cs. Tóth, C. G. R. Geddes, E. Esarey, and W. P. Leemans \\ Lawrence Berkeley National Laboratory, University of California, Berkeley, California 94720, USA
}

Received August 17, 2006; revised October 26, 2006; accepted October 26, 2006; posted October 30, 2006 (Doc. ID 74161); published January 12, 2007

The electric field profiles of broad-bandwidth coherent terahertz (THz) pulses, emitted by laser-wakefieldaccelerated electron bunches, are studied. The near-single-cycle $\mathrm{THz}$ pulses are measured with two singleshot techniques in the temporal and spatial domains. Spectra of $0-6 \mathrm{THz}$ and peak fields up to $\simeq 0.4 \mathrm{MV} \mathrm{cm}^{-1}$ are observed. The measured field substructure demonstrates the manifestation of spatiotemporal coupling at focus, which affects the interpretation of $\mathrm{THz}$ radiation as a bunch diagnostic and in highfield pump-probe experiments. () 2007 Optical Society of America

OCIS codes: $020.1670,140.7090,320.5540$.

Intense terahertz $(\mathrm{THz})$ radiation, covering electromagnetic wavelengths of $10-1000 \mu \mathrm{m}$, is interesting for studies of ultrafast processes in semi- and superconductors. Peak THz fields of the order of $\mathrm{MV} \mathrm{cm}^{-1}$ allow for use of the $\mathrm{THz}$ pulse as the pump beam in pump-probe experiments. ${ }^{1}$ While conventional laserbased sources (e.g., optical rectification or a photoconductive antenna) are typically limited to generation of $\approx 50 \mathrm{kV} \mathrm{cm}^{-1}$ fields, coherent $\mathrm{THz}$ emission from femtosecond electron bunches has been described to yield single-cycle radiation that is potentially 1 to 2 orders of magnitude more intense. ${ }^{2,3}$ The coherent $\mathrm{THz}$ radiation from such sources is also used as a temporal electron bunch diagnostic. ${ }^{2,4}$ The results described in this Letter are based on $\mathrm{THz}$ emission from electron bunches produced by a laser wakefield accelerator $^{5}$ (LWFA). The LWFA delivers multinanocoulomb relativistic electron bunches, intrinsically synchronized to the laser pulse. Through emission of coherent transition radiation (CTR), these ultrashort bunches emit $\mathrm{THz}$ pulses as they exit the plasma-vacuum boundary. ${ }^{3-5}$

Studies of spatiotemporal field coupling ${ }^{6,7}$ at focus have been performed with scanning techniques on conventional low-field laser-based $\mathrm{THz}$ sources. 8 Here we report on single-shot measurements of the temporal and spatial profiles of intense LWFAproduced $\mathrm{THz}$ pulses. The spatiotemporal coupling impacts the temporal properties of the radiation pulse and, therefore, the interpretation of LWFAbased experimental results. In addition, due to fluctuations in the LWFA performance, development of single-shot techniques is required.

The high-power Ti: $\mathrm{Al}_{2} \mathrm{O}_{3}$ laser of the Lasers, Optics and Accelerator Systems Integrated Studies (LOASIS) facility ${ }^{5}$ at the Lawrence Berkeley National Laboratory was used for the experiments. In the experiment, an electron bunch with a total charge of $\simeq 2 \mathrm{nC}$ was produced, while its energy distribution $g(E)$ was measured to be of the form $g(E)$ $\propto \exp \left(-E / E_{t}\right)$, with $E_{t}=5 \mathrm{MeV}$. $\mathrm{THz}$ radiation was emitted as the electrons propagated through the dielectric discontinuity of the plasma-vacuum boundary. ${ }^{3-5}$ Two variations of the electro-optic sampling (EOS) technique were operated to characterize the LWFA-produced $\mathrm{THz}$ pulses, namely, a singleshot temporal cross-correlation technique ${ }^{10,11}$ and a single-shot two-dimensional (2D) spatial technique. ${ }^{12}$

An $f / 290^{\circ}$ off-axis parabola (OAP2, $15 \mathrm{~cm}$ focal length), positioned off center $\left(\theta=19^{\circ}\right.$ with respect to the main axis), was used to collect and collimate a portion of the emitted $\mathrm{THz}$ radiation (see Fig. 1). The collimated $\mathrm{THz}$ radiation was focused by an $f / 2.490^{\circ}$ off-axis parabola (OAP3, $18 \mathrm{~cm}$ focal length) onto a $200 \mu \mathrm{m}$ thick GaP crystal outside the target chamber [through a polyethylene (PE) window].

For the single-shot temporal EOS technique, two laser beams, both with a minimum pulse length of $70 \mathrm{fs}$ (intensity FWHM), were split off from the main laser beam, conserving synchronization. The first probe beam, $I_{1}(t)$ in Fig. 1, was stretched (chirped) to a length of $1350 \mathrm{fs}$ (intensity FWHM). After propagation through a polarizer, the laser pulse was focused (spot size $<20 \mu \mathrm{m})$ to overlap with the $\mathrm{THz}$ beam in

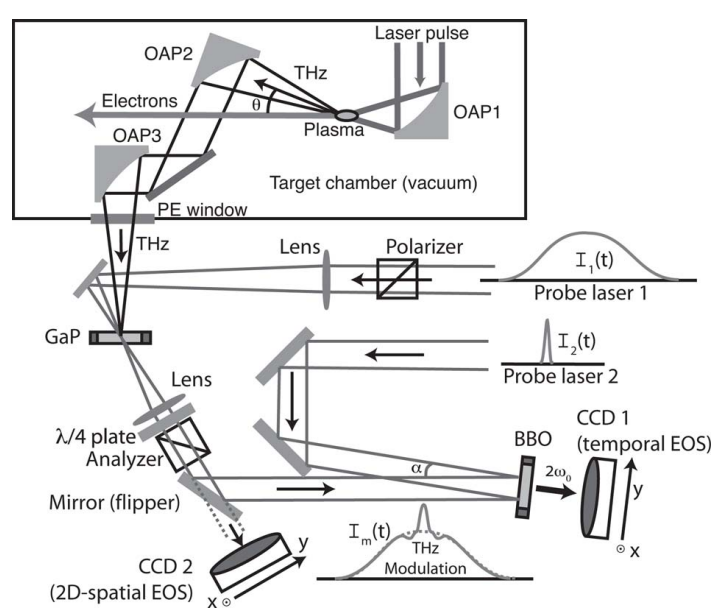

Fig. 1. Schematic representation of the LWFA, the THz (CTR) beam path, and the two EOS detection setups (temporal and $2 \mathrm{D}$ spatial). Abbreviations defined in text. 
the (110)-cut GaP. A $\lambda / 4$ plate was rotated to yield a circular polarization state incident on the analyzer.

Through the $\mathrm{THz}$-induced electro-optic (EO) effect in the GaP crystal, the intensity envelope of probe laser $I_{1}(t)$, transmitted through the analyzer, was modulated to $I_{m}(t)$. It can be shown ${ }^{13}$ that $I_{m}(t)$ $=1 / 2\left[1+\sin \Gamma^{*}(t)\right] I_{1}(t)$, where in the Fourier domain $\Gamma^{*}(\nu)=\Gamma_{\mathrm{THz}}(\nu) T_{\mathrm{GaP}}(\nu)$ and $\nu$ is the radiation frequency. $\Gamma^{*}(\nu)$ represents the THz-induced phase retardation and is a convolution of the source profile $\Gamma_{\mathrm{THz}}(\nu)$ and crystal effects $T_{\mathrm{GaP}}(\nu){ }^{13,14}$ The function $T_{\mathrm{GaP}}(\nu)$ for $200 \mu \mathrm{m}$ thick GaP has previously been discussed $^{15}$; over the frequency range $0-8 \mathrm{THz}$, dispersion effects were found to be relatively limited. $\Gamma_{\mathrm{THz}}(t)$ is related to the original $\mathrm{THz}$ field profile $E_{\mathrm{THz}}(t)$ through $^{14} \Gamma_{\mathrm{THz}}(t)=2 \pi L n_{0}^{3} r_{41} E_{\mathrm{THz}}(t) / \lambda_{0}$, with $L=200 \mu \mathrm{m}$ the GaP crystal thickness, $n_{0}=3.19$ the index of refraction in $\mathrm{GaP}$ at $\lambda_{0}$, and $r_{41} \simeq 2$ $\times 10^{-12} \mathrm{mV}^{-1}$ the EO coefficient of GaP. ${ }^{16}$ For CTR, the $\mathrm{THz}$ field $E_{\mathrm{THz}}(\nu)$ is determined by the Fourier transformation of the electron bunch charge profile and diffraction effects from the limited size of the transverse plasma-vacuum interface. ${ }^{3,15}$

The EO-modulated laser envelope was recorded in a single-shot manner ${ }^{10}$ through noncollinear sumfrequency generation of $I_{m}(t)$ and a second short laser pulse $I_{2}\left(t-\tau_{2}\right)$ (pulse length of $70 \mathrm{fs}$ FWHM, which determines the time resolution of the system), with $\tau_{2}$ the respective temporal delay between both laser beams. The frequency doubling occurred in a $\beta$-barium borate (BBO) crystal. A CCD camera [CCD 1 (see Fig. 1)] recorded the time-integrated transverse ( $x y$-plane) intensity distribution of the frequency-doubled radiation $I_{2 \omega}(x, y)$. During postprocessing, each image $I_{2 \omega}(x, y)$ was integrated over $x$ to yield $I_{2 \omega}(y)$. Also, each profile was normalized to yield $I_{2 \omega} \rightarrow\left(I_{2 \omega}-I_{2 \omega, 0}\right) / I_{2 \omega, 0}$, with $I_{2 \omega, 0}$ the reference profile in absence of a THz pulse. Due to the noncollinear geometry, the delay $\tau_{2}$ is a function of the position $y$ within the crystal and $I_{2 \omega}(y)$ can be converted to a temporal profile $I_{2 \omega}\left(\tau_{2}\right)$ through $\tau_{2}$ $=(y / c) \tan \left(\alpha^{*} / 2\right)$, with $c$ the speed of light in vacuum. The angle of incidence between both laser pulses at the crystal surface was $\alpha=10^{\circ}$ (inside the BBO crystal $\alpha^{*}=6.3^{\circ}$ ), and the laser beam diameters were $\simeq 6 \mathrm{~mm}$ (intensity $\mathrm{FWHM}$ ). After defining a measured retardation function as $\Gamma_{\text {cor }}\left(\tau_{2}\right)=\arcsin I_{2 \omega}\left(\tau_{2}\right)$, it can be found ${ }^{13,14,17}$ that

$$
\Gamma_{\text {cor }}(\nu) \simeq \Gamma_{\mathrm{THz}}(\nu) T_{\mathrm{GaP}}(\nu) I_{\mathrm{env}, 2}(\nu),
$$

with $I_{\mathrm{env}, 2}(\nu)$ the envelope spectrum of $I_{2}(t)$.

Three measured representative phase retardation profiles $\Gamma_{\text {cor }}(t)$ and $\left|\Gamma_{\text {cor }}(\nu)\right|$, obtained through the single-shot measurement of $I_{2 \omega}\left(\tau_{2}\right)$, are shown in Figs. 2(a) and 2(b), respectively. Shot A in Fig. 2(a) shows a field profile with just one main field cycle (spectrum extending to $\simeq 6 \mathrm{THz}$ ) and peak phase retardation of $\Gamma_{\text {cor }} \simeq 1 \mathrm{rad}$. Through relation (1), and considering that the field reducing effects of dispersion and surface transmission in the GaP crystal can be approximated as $T_{\mathrm{GaP}} \simeq 0.25,{ }^{15}$ an estimation of
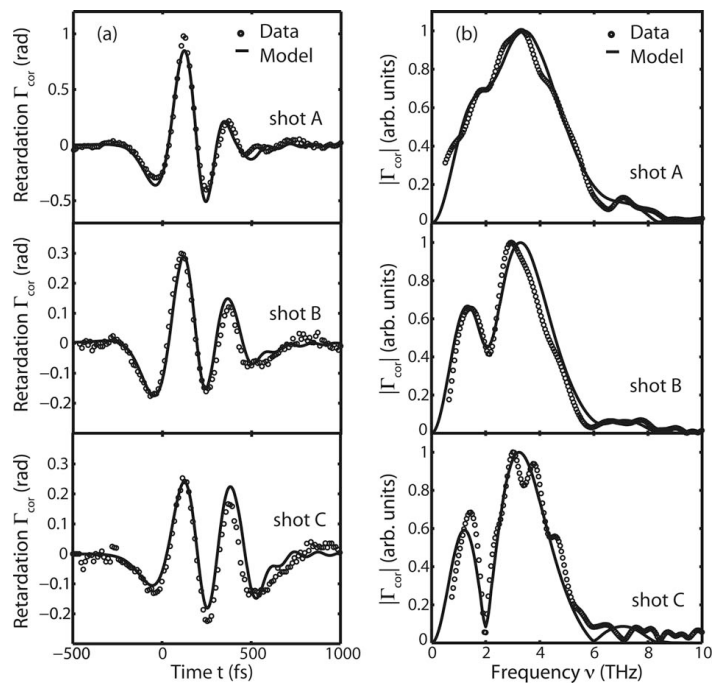

Fig. 2. (a) Measured single-shot $\mathrm{THz}$ field profiles in terms of phase retardation $\Gamma_{\text {cor }}(t)$. (b) Fourier transformation $\left|\Gamma_{\text {cor }}(\nu)\right|$ of the data. The solid curves are calculated from a CTR-based model.

the $\mathrm{THz}$ field amplitude at the crystal location yields $E_{\mathrm{THz}} \simeq 0.4 \mathrm{MV} \mathrm{cm}^{-1}$. The estimated sensitivity (based on fluctuation of $I_{2 \omega, 0}$ ) is $\simeq 20 \mathrm{kV} \mathrm{cm}^{-1}$.

Figure 2 also displays the presence of a trailing $\mathrm{THz}$ pulse (two maxima in the temporal domain and interference in the spectral domain, more clearly visible in shots B and C). Although the position of the trailing pulse was relatively stable at a delay of 230-250 fs, its relative field strength showed strong fluctuations (from weaker in shot A to stronger in shot C). Each measurement (in both domains) was compared with modeled ${ }^{4,15}$ EOS profile, which yielded best agreement if a $45 \mathrm{fs}$ (rms) electron bunch was assumed (one bunch emitting two $\mathrm{THz}$ pulses at a separation of $240 \mathrm{fs}$ ). The relative field amplitude of the modeled trailing pulse was $30 \%, 57 \%$, and $90 \%$ for shots A, B, and C, respectively.

For single-shot 2D-spatial $\mathrm{THz}$ imaging, the setup in Fig. 1 was slightly modified. The $\lambda / 4$ plate and the two lenses in the path of probe laser 1 were removed, such that a collimated laser beam was overfilling the $\mathrm{THz}$ spot at the GaP crystal. By removing the flippermirror, the $2 \mathrm{D}$ time-integrated intensity distribution $I_{2 \mathrm{D}}(x, y, \tau)$ was recorded by CCD camera 2 , with $\tau$ the delay between $E_{\mathrm{THz}}(t)$ and $I_{1}(t)$. The compressor for probe laser 1 was tuned to yield a pulse length of $70 \mathrm{fs}$ (intensity FWHM), which allowed for timeresolved 2D imaging. For a linear-polarized laser beam, it can be derived ${ }^{13,14}$ that $I_{2 \mathrm{D}}(x, y, \tau) \propto \int I_{1}(t$ $-\tau) \sin ^{2}\left[\Gamma^{*}(x, y, t) / 2\right] \mathrm{d} t$, with $\Gamma^{*}(\nu)$ previously defined in the Fourier domain.

After switching the setup to the single-shot 2D EOS technique, the 2D laser transmission $I_{2 \mathrm{D}}(x, y, \tau)$ was recorded while varying the delay $\tau$ between the laser and $\mathrm{THz}$ beam. It was found that the $2 \mathrm{D} \mathrm{THz}$ profile was stable at a given delay, but evolved over time. Three characteristic $I_{2 \mathrm{D}}(x, y, \tau)$ images are shown in Figs. 3(a)-3(c), with (a) $\tau=-250 \mathrm{fs}$, (b) $\tau$ 
$=0 \mathrm{fs}$, and (c) $\tau=350 \mathrm{fs}$. The data show that a main $\mathrm{THz}$ spot is present [see, e.g., Fig. 3(b)], where the main spot has an intensity FWHM of $\simeq 600 \mu \mathrm{m}$. The substructure indicates the presence of effects from diffraction [e.g., shot D in Fig. 3(a)], coma [e.g., shot F in Fig. 3(c)], and other aberrations. Diffraction is present because the $\mathrm{THz}$ emission overfills parabola OAP2 (see Fig. 1), while coma can be present if the alignment of OAP2 and OAP3 is not perfect. At a fixed delay $\tau=0 \mathrm{fs}$, another series of images was analyzed by taking lineouts at $y=0 \mathrm{~mm}$; several lineouts, shown in Fig. 4, indicate that the position of the substructure is stable, but that the relative intensity fluctuates shot to shot.

It was found that it is unlikely that the doubleTHz-pulse structure (e.g., EOS measurements in Fig. 2 ) is a result of the LWFA production of two electron bunches as previously hypothesized. ${ }^{15}$ The absence of a correlation between the THz-pulse features with LWFA parameters such as electron energy and plasma density supports this conclusion. The doubleTHz-pulse profile is rather the result of optical effects in the $\mathrm{THz}$ focusing system (diffraction, coma, and other aberrations). A heuristic ray-based model (excluding effects such as the Gouy shift and bandwidthinduced dispersion at focus), where each ray was defined as a single-cycle pulse with a finite transverse dimension, was developed and confirmed comainduced occurrence of a double-pulse profile. ${ }^{17} \mathrm{Ex}-$ perimentally, the spatiotemporal coupling is fixed by the arrangement and alignment of the optics in the $\mathrm{THz}$ beam line, resulting in a stable positioning of the temporal and spatial substructure (see Figs. 2 and 4). However, fluctuations of the electron bunch energy and pointing can result in a varying angular $\mathrm{THz}$ emission profile, which yields fluctuations in the amplitude ratio of the field structure.
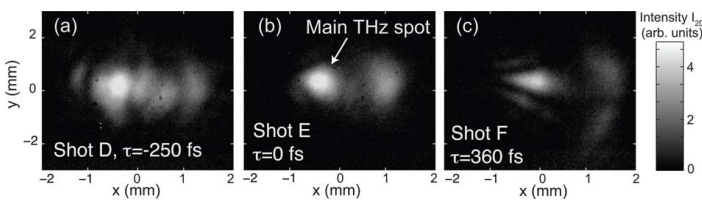

Fig. 3. (Color online) Three representative single-shot 2D $\mathrm{THz}$ images $I_{2 \mathrm{D}}(x, y, \tau)$, taken at different values for the delay $\tau$ between the $\mathrm{THz}$ and laser pulse.

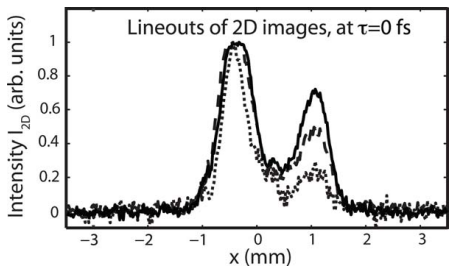

Fig. 4. At a fixed delay $\tau=0 \mathrm{fs}$, lineouts at $y=0$ of three consecutive $2 \mathrm{D}$ shots are displayed.
In summary, single-cycle broad-bandwidth $\mathrm{THz}$ pulses were studied at focus, demonstrating the production of $\mathrm{THz}$ pulses with spectra of $0-6 \mathrm{THz}$ and peak fields of $\simeq 0.4 \mathrm{MV} \mathrm{cm}^{-1}$. A trailing $\mathrm{THz}$ pulse, with varying amplitude shot to shot, was observed at a stable separation of $\simeq 240 \mathrm{fs}$. A $2 \mathrm{D}$-spatial EOS technique indicated the presence of a main $\mathrm{THz}$ spot surrounded by time-evolving substructure. Both techniques support the conclusion that diffraction, coma, and other aberrations are spatiotemporally coupled in the focal volume.

This work was supported by the U.S. Department of Energy under contract DE-AC02-05CH11231. J. van Tilborg's e-mail address is JvanTilborg@lbl.gov.

\section{References}

1. M. S. Sherwin, C. A. Schmuttenmaer, and P. H. Bucksbaum, eds., Opportunities in $\mathrm{THz}$ Science (DOENSF-NIH workshop, 2004).

2. G. L. Carr, M. C. Martin, W. R. McKinney, K. Jordan, G. R. Neil, and G. P. Williams, Nature 420, 153 (2002).

3. C. B. Schroeder, E. Esarey, J. van Tilborg, and W. P. Leemans, Phys. Rev. E 69, 016501 (2004).

4. J. van Tilborg, C. B. Schroeder, C. V. Filip, Cs. Tóth, C. G. R. Geddes, G. Fubiani, R. Huber, R. A. Kaindl, E. Esarey, and W. P. Leemans, Phys. Rev. Lett. 96, 014801 (2006).

5. W. P. Leemans, C. G. R. Geddes, J. Faure, Cs. Tóth, J. van Tilborg, C. B. Schroeder, E. Esarey, G. Fubiani, D. Auerbach, B. Marcelis, M. A. Carnahan, R. A. Kaindl, J. Byrd, and M. C. Martin, Phys. Rev. Lett. 91, 074802 (2003).

6. M. Kempe and W. Rudolph, Phys. Rev. A 48, 4721 (1993).

7. M. A. Porras, Phys. Rev. E 65, 026606 (2002).

8. S. Hunsche, S. Feng, H. G. Winful, A. Leitenstorfer, M. C. Nuss, and E. P. Ippen, J. Opt. Soc. Am. A 16, 2025 (1999).

9. Z. Jiang and X.-C. Zhang, Opt. Express 5, 243 (1999).

10. S. P. Jamison, J. Shen, A. M. MacLeod, W. A. Gillespie, and D. A. Jaroszynski, Opt. Lett. 28, 1710 (2003).

11. S. P. Jamison, G. Berden, A. M. MacLeod, D. A. Jaroszynski, B. Redlich, A. F. G. van der Meer, and W. A. Gillespie, Nucl. Instrum. Methods Phys. Res. A 557, 305 (2006).

12. Q. Wu, T. D. Hewitt, and X.-C. Zhang, Appl. Phys. Lett. 69, 1026 (1996)

13. G. Gallot and D. Grischkowsky, J. Opt. Soc. Am. B 16, 1204 (1999).

14. J. Faure, J. van Tilborg, R. A. Kaindl, and W. P. Leemans, Opt. Quantum Electron. 36, 681 (2004).

15. J. van Tilborg, C. B. Schroeder, C. V. Filip, Cs. Tóth, C. G. R. Geddes, G. Fubiani, E. Esarey, and W. P. Leemans, Phys. Plasmas 13, 056704 (2006).

16. Q. Wu and X.-C. Zhang, Appl. Phys. Lett. 70, 1784 (1997).

17. J. van Tilborg, "Coherent terahertz radiation from laser-wakefield-accelerated electron beams," Ph.D. thesis (Eindhoven University of Technology, 2006). 